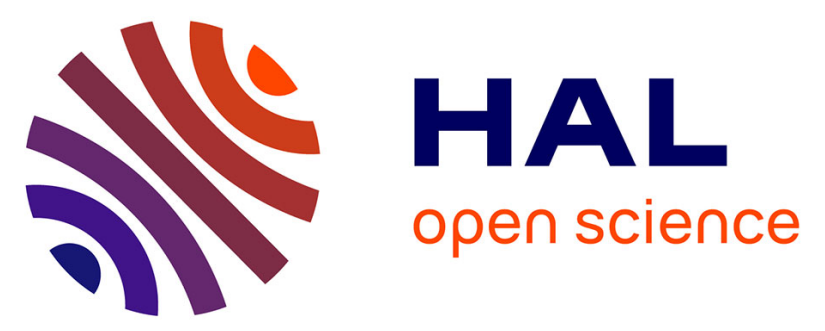

\title{
Quantitative full-colour transmitted light microscopy and dyes for concentration mapping and measurement of diffusion coefficients in microfluidic architectures
}

Martinus H. V. Werts, Vincent Raimbault, Rozenn Texier-Picard, Rémi

Poizat, Olivier Français, Laurent Griscom, Julien R. G. Navarro

\section{To cite this version:}

Martinus H. V. Werts, Vincent Raimbault, Rozenn Texier-Picard, Rémi Poizat, Olivier Français, et al.. Quantitative full-colour transmitted light microscopy and dyes for concentration mapping and measurement of diffusion coefficients in microfluidic architectures. Lab on a Chip, 2012, 12, pp.808820. $10.1039 / \mathrm{c} 2 \mathrm{lc} 20889 \mathrm{j}$. hal-00662353

\section{HAL Id: hal-00662353 \\ https://hal.science/hal-00662353}

Submitted on 27 Jul 2015

HAL is a multi-disciplinary open access archive for the deposit and dissemination of scientific research documents, whether they are published or not. The documents may come from teaching and research institutions in France or abroad, or from public or private research centers.
L'archive ouverte pluridisciplinaire HAL, est destinée au dépôt et à la diffusion de documents scientifiques de niveau recherche, publiés ou non, émanant des établissements d'enseignement et de recherche français ou étrangers, des laboratoires publics ou privés. 


\section{Quantitative full-colour transmitted light microscopy and dyes for concentration mapping and measurement of diffusion coefficients in microfluidic architectures}

Martinus H. V. Werts, ${ }^{a, b}$ Vincent Raimbault, ${ }^{c}$ Rozenn Texier-Picard, ${ }^{a, d}$ Rémi Poizat, ${ }^{a, b}$ Olivier Français, ${ }^{\mathrm{b}, \mathrm{e}}$ Laurent Griscom, ${ }^{\mathrm{a}, \mathrm{b}}$ and Julien R. G. Navarro ${ }^{\mathrm{a}, \mathrm{b}}$

a Ecole Normale Supérieure de Cachan-Bretagne, UEB, Campus de Ker Lann, F-35170 Bruz, France

${ }^{\mathrm{b}}$ CNRS, SATIE (UMR8029), ENS Cachan-Bretagne, UEB, Campus de Ker Lann, F-35170 Bruz, France

' CNRS, IMS (UMR5218), Université de Bordeaux, 351 cours de la Libération, F-33405 Talence Cedex, France

${ }^{\mathrm{d}}$ CNRS, IRMAR (UMR6625), ENS Cachan-Bretagne, UEB, Campus de Ker Lann, F-35170 Bruz, France

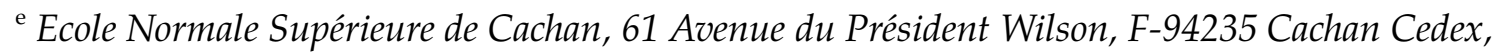
France

* corresponding author

Author manuscript for:

Lab on Chip 2012, 12, 808-820

DOI: $10.1039 /$ C2LC20889J

\section{Supplementary information}

Electronic supplementary information (ESI) available. See DOI: 10.1039/c2lc20889j 


\section{Table of contents graphic}

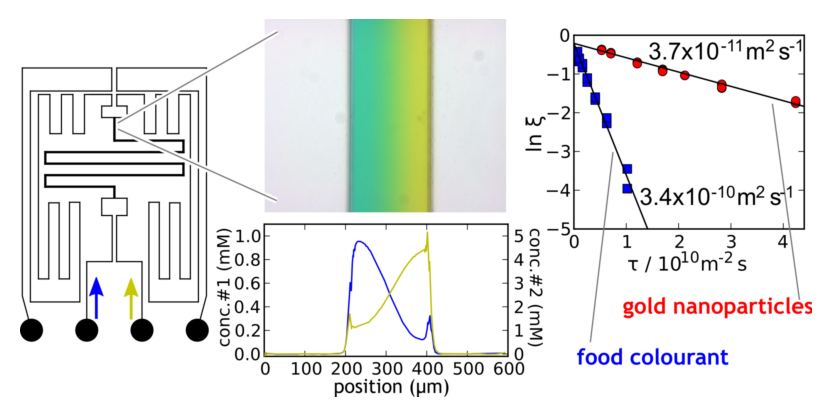

\section{Abstract}

A simple and versatile methodology has been developed for the simultaneous measurement of multiple concentration profiles of colourants in transparent microfluidic systems, using conventional transmitted light microscopy, a digital colour (RGB) camera and numerical image processing combined with multicomponent analysis. Rigourous application of BeerLambert law would require monochromatic probe conditions, but in spite of the broad spectral bandwidths of the three colour channels of the camera, a linear relation between the measured optical density and dye concentration is established under certain conditions. An optimised collection of dye solutions for the quantitative optical microscopic characterisation of microfluidic devices is proposed. Using the methodology for optical concentration measurement we then implement and validate a simplified and robust method for the microfluidic measurement of diffusion coefficients using an H-filter architecture. It consists in measuring the ratio of the concentrations of the two output channels of the H-filter. It enables facile determination of the diffusion coefficient, even for non-fluorescent molecules and nanoparticles, and is compatible with non-optical detection of the analyte. 


\section{Introduction}

Solutions of food dyes or biological stains are frequently used to visualise fluidic channels in transparent microsystems using transmitted light microscopy. In most cases these dyes are used for qualitative purposes, although they have been used for quantitative measurements, such as in situ channel height ${ }^{1}$ and concentration profiles. ${ }^{2}$ Offline UV-visible absorbance measurements have been used for microfluidic flow rate determination. ${ }^{3}$ Quantitative measurements of concentration profiles ${ }^{4-7}$ in microfluidics are mostly achieved using fluorescence imaging and fluorescent probes which requires sensitive detectors and suitable microscope optics and hardware.

Previous work on absorbance imaging in transmitted light microscopy used narrow-band light sources (either via monochromators or band pass filters) in combination with monochrome cameras. ${ }^{1,28-13}$ Conventional transmitted light microscopes are readily equipped with colour RGB (red-green-blue) cameras. One goal of this work is to establish to what extent full-colour transmitted light imaging using RGB cameras can be combined with suitable dye solutions to enable the simultaneous measurement of multi-component concentration distributions. The spectral bands covered by each of the colour channels are rather wide (> $100 \mathrm{~nm}$ ), which is at odds with rigourous application of Beer-Lambert law that requires monochromatic light. ${ }^{14}$ In the present work it is shown that within certain limits Beer-Lambert law can still be applied to relate transmitted light (RGB) intensities to dye concentrations.

The choice of dyes for use in microfluidics is vast, many are available in different formulations. Optimised protocols describing recommended concentrations, solution composition etc. for microfluidics are not readily available. This may lead to a number of less desirable situations such as the use of unnecessarily high dye concentrations or the use of inks of unknown proprietary composition. Here we study the properties of a small collection of organic dyes and their application in quantitative studies of microfluidic architectures, in order to establish a versatile collection of complementary dye solutions of known and welldefined composition. 
When calibrated, quantitative measurements using these dyes in transmitted light microscopy are indeed possible. In this paper, conditions are established for the measurement of multicomponent concentration profiles using conventional transmitted light microscopes equipped with suitably adjusted RGB cameras. In view of the use of nonmonochromatic detection, it is necessary to verify linearity of the absorbance signal for each dye. Moreover, the additivity of the absorbance signals for two dyes needs to be checked in the case of multicomponent detection. The methodology presented can naturally be applied to other dyes than those used in this paper, and we demonstrate the relevance of the method for concentration mapping of $13 \mathrm{~nm}$ diameter gold nanoparticles diffusing in a microfluidic circuit.

Building on transmitted-light based concentration measurements, we propose a microfluidic method for the measurement of diffusion coefficients which is a modification of the Tsensor/H-filter architectures ${ }^{15-19}$ often used for studying the molecular diffusion of fluorescent objects. The alternative method presented here enables the measurement of diffusion coefficients of nonfluorescent species using transmitted light microscopy, with the further possibility to even use non-optical methods for detecting the diffusing species. The method is applied to the (mostly non-fluorescent) food dyes studied as well as to (nonfluorescent) gold nanoparticles.

Finally, we illustrate the use of quantitative full colour transmitted light microscopy in the characterisation of typical microfluidic mixers and gradient generators. Not only do optimised dye solutions and optimised image acquisition immediately generate visually pleasing photographs, they also render these images amenable to multicomponent concentration measurements that can reveal minor details in the functioning of microfluidic devices not immediately recognisable in the initial images.

\section{Dyes and solutions}

\subsection{Materials}

As a solvent for the studies in this work we exclusively use phosphate-buffered saline solution (PBS; $10 \mathrm{mM}$ phosphate buffer at pH 7.4 with $137 \mathrm{mM} \mathrm{NaCl}$ and $2.7 \mathrm{mM} \mathrm{KCl}$ in water) as this provides a constant physicochemical environment for all dyes, allowing 
multiple dyes to be used simultaneously without a mismatch in either $\mathrm{pH}$ or ionic strength. Pure water has an unstable $\mathrm{pH}$ under ambient conditions. ${ }^{20}$ Additionally, ionic strength is an important physicochemical parameter. Most dyes used here are not sensitive to $\mathrm{pH}$ within the $\mathrm{pH}$ range 5-9, and many will very likely give consistent results when used with pure water, provided that they are used as their water-soluble salts. However, when possible a saline buffer such as PBS is the preferred choice. PBS solutions were prepared from preformulated powder in individual pouches (Sigma/Aldrich). It is noted that in the microfluidic literature PBS (phosphate buffer containing also $\mathrm{NaCl}$ and $\mathrm{KCl}$ ) is sometimes unfortunately confused with simple phosphate buffer (without additional salts).

The dyes were obtained as pure powders and used as received. The dyes chosen are either food dyes or stains for biological microscopy (or both). Some of the food dyes may be obtained in solution from grocery stores as food coloring, but these preparations contain several additives such as propylene glycol and preservatives, and are of unknown concentration. We did not compare these preparations with the PBS solutions of the pure dyes used in this work. Although - depending on the local legislation - most dyes are considered to be safe when used as a food additive in a dilute form, the pure powders should be handled with care, since these are the dye in high dose, and some are considered irritants, and even poisons at high concentration.

The choice of dyes was inspired by a survey of microfluidic literature (see e.g. Refs. 21 and 22), chemical experience and commercial availability. One particular goal was to obtain a combination of three dyes corresponding to colours complementary to red, green, and blue, i.e. cyan, magenta and yellow, as this will enable the best colour separation between the three dyes in a colour camera. Overlapping colour responses, however, is not necessarily a problem, as analytical chemical multicomponent analysis methodology is capable of transforming such overlapping responses into individual component concentrations.

The identification of suitable yellow (Tartrazine) and cyan (Fast Green) dyes was straightforward, but finding the best water-soluble magenta dye proved more difficult, and this explains the preponderance of red-magenta in the selection of dyes studied. Dyes were chosen from the family of traditional food dyes (water-soluble azo dyes and related compounds), but we also selected several xanthene dyes (fluorescein and derivatives). 
Tartrazine (food additive E102, FD\&C Yellow \#5, CAS: 1934-21-0, mol. wt. $534.4 \mathrm{~g} \mathrm{~mol}^{-1}$ ) is a yellow water-soluble negatively charged azo dye. Amaranth (food additive E123, FD\&C Red No. 2, CAS: $915-67-3$, mol.wt. $604.5 \mathrm{~g} \mathrm{~mol}^{-1}$ ) is a red-magenta dye, also of the azo dye family. In some countries it has been discontinued as a food dye, but is still commercially available and useful as a microfluidic dye. Allura Red AC (food additive E129, FD\&C Red \#40, CAS: 25956-17-6, mol. wt. $496.4 \mathrm{~g} \mathrm{~mol}^{-1}$ ) is a red azo dye. It is sometimes used as a replacement food dye for Amaranth (E123), with a hue that is red instead of magenta. Fast Green FCF (food additive E143, FD\&C Green \#3, CAS: 2353-45-9, mol. wt. $808.9 \mathrm{~g} \mathrm{~mol}^{-1}$ ) is a watersoluble triaryl dye which produces a cyan (blue-green) colour in PBS solution. In combination with yellowish substances it produces a vivid green colour.

Fluorescein, free acid (no food dye designation, CAS: 2321-07-5, mol. wt. $332.3 \mathrm{~g} \mathrm{~mol}^{-1}$ ) Fluorescein was also included in the study, since it can be used both in transmitted light as a blue-absorbing yellow dye and in fluorescence measurements. It has been approved for injection into the blood stream of humans, in particular for ocular angiography. Confusion exists concerning the type of fluorescein to be used as a fluorescent tracer in fluidic channels. Sometimes, fluorescein isothiocyanate (FITC) is used instead of fluorescein, with the implicit incorrect assumption that these compounds are interchangeable. Fluorescein and FITC are different compounds with different chemical and photophysical properties. In particular, FITC readily hydrolyses in aqueous solution and is converted rapidly into aminofluorescein. An aqueous solution of FITC is thus actually a solution of aminofluorescein, which has different photophysical characteristics than fluorescein. For these reasons, it is preferable to use fluorescein as a tracer rather than FITC. The use of the latter compound should be reserved for its intended use, i.e. fluorescent labeling of (bio)macromolecules (with subsequent purification of the labeled macromolecules).

Fluorescein - like most other xanthene dyes (such as Erythrosine and Rose Bengal) is sold in various forms, in particular as the free acid or as its disodium salt. We used the free acid, since there is a better guarantee of high purity. A disadvantage of the free acid is that its dissolution in PBS, and even in aqeuous $\mathrm{NaOH}$ is slow. In order to be solubilised, the (initially solid) free acid needs to be deprotonated, and this solid-liquid process is not fast. Extensive sonication (1h) in PBS eventually leads to a limpid solution. 
Erythrosin B, free acid (CAS: 15905-32-5, $834.9 \mathrm{~g} \mathrm{~mol}^{-1}$ ) is a red-magenta tetra-iodinated derivative of fluorescein, and it is fluorescent (green-yellow), although its fluorescence quantum yield is significantly lower than that of fluorescein. Its disodium salt is known as food additive E127 or FD\&C Red \#3. Studies have shown that significant dye aggregation (> $10 \%$ of the dye molecules) occurs in water at concentration above $0.5 \mathrm{mM}$ leading to altered photophysical characteristics. ${ }^{23}$ Therefore, higher concentrations of the dye were avoided.

Rose Bengal (no food dye designation, CAS: 632-69-9, $1018 \mathrm{~g} \mathrm{~mol}^{-1}$ ) is also a magenta derivative of fluorescein and is used as a biological stain in microscopy, as a photoinitiator for photopolymerisation $^{24}$ and as a photosensitiser for the generation of singlet oxygen (of interest both in synthetic chemistry and in photodynamic therapy). ${ }^{25}$ At concentration above $0.5 \mathrm{mM}$, significant aggregation of the dye occurs. ${ }^{23}$

As a complement to organic dyes we studied solutions of $13 \mathrm{~nm}$ diameter gold nanoparticles capped $^{26}$ with thiooctic acid (AuTA). Gold and silver nanoparticles have an extremely strong optical response related to the localised plasmonic resonance of their conduction electrons. ${ }^{27}$ This optical response manifests itself as visible light absorption, leading to burgundy red coloration of gold nanoparticles, and - for particles of sufficient diameter - efficient resonant light scattering. ${ }^{28,29}$ In combination with the possibility to functionalise these metal particles with (bio)molecular functions ${ }^{30,31}$ the strong optical response makes gold and silver particles interesting biophotonic transducers for sensitive detection in microfluidic volumes. The solutions of AuTA nanoparticles used here are easily prepared, are stable against aggregation (at basic $\mathrm{pH}$ ) and can readily be concentrated to the concentrations necessary for observation in microfluidic channels using transmitted light optical microscopy. The particles, however, are not soluble in PBS solution, and therefore $1 \mathrm{mM}$ aqueous $\mathrm{NaOH}$ was used as a solvent instead.

\subsection{Absorption spectra and extinction coefficients}

Figure 1 displays the UV-visible absorption spectra of PBS solutions of the dyes. These spectra illustrate their spectral complementarity, particularly of Tartrazine, Amaranth (or Rose Bengal) and Fast Green. The wavelengths of the absorption maxima and the corresponding extinction coefficients are listed in Table 1. These parameters are of use for offline analysis of dye solutions. The magnitude of the extinction coefficients reveals the three 
distinct chemical families of the dyes: azo dyes $\left(\varepsilon_{\max } \sim 2 \times 10^{4} \mathrm{M}^{-1} \mathrm{~cm}^{-1}\right)$, xanthene dyes $\left(\varepsilon_{\max } \sim\right.$ $\left.8 \times 10^{4} \mathrm{M}^{-1} \mathrm{~cm}^{-1}\right)$ and Fast Green triarylmethane dye $\left(\varepsilon_{\max }>10^{5} \mathrm{M}^{-1} \mathrm{~cm}^{-1}\right)$. For AuTA we list the typical literature value for $13 \mathrm{~nm}$ diameter gold nanoparticles, which should be taken to be accurate within $\pm 20 \%{ }^{32}$ The high extinction coefficient for these particles makes that even the concentrated solutions used for the microfluidic experiments reported here have a particle concentration over a thousand times smaller than the dye solutions.
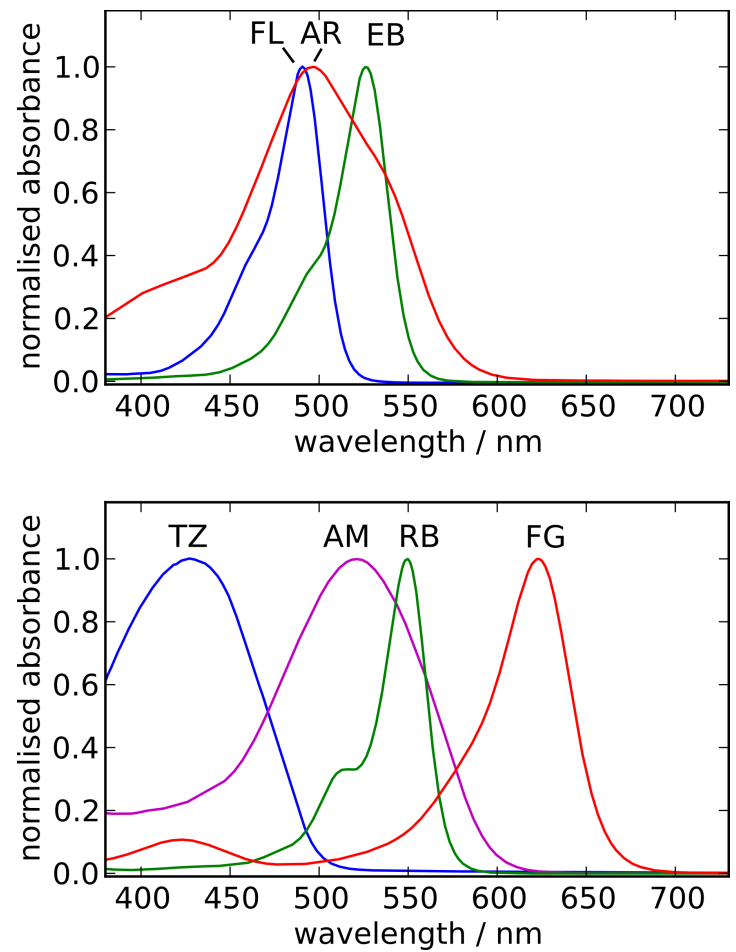

Figure 1. Normalised UV-visible absorption spectra of solutions of dyes in PBS solution. $F L=$ Fluorescein, $A R=$ Allura Red $A C, E B=$ erythrosine $B, T Z=$ tartrazine, $A M=$ Amaranth,$R B=$ Rose Bengal, FG = Fast Green FCF.

Table 1 also list the maximum recommended concentrations and the recommended concentration of the dyes in PBS for use with a $100 \mu \mathrm{m}$ high fluidic channel. The maximum concentration was either limited to the solubility of the dye or to $4 \mathrm{mM}$. No concentrations higher than $4 \mathrm{mM}$ were tested, in view of the PBS solution being only $10 \mathrm{mM}$ in buffering phosphate. For the azo dyes the $4 \mathrm{mM}$ concentration does not seem to be a limit, but using these concentrations would need a change of buffer (e.g. $0.1 \mathrm{M}$ phosphate buffer at $\mathrm{pH} 7.4$ ) and additional validation. Except for Fast Green, which has some headroom, the concentrations for use with $100 \mu \mathrm{m}$ are limited by the maximum recommended concentration. For use with thicker channels, the solutions should be diluted proportionally. 
Thinner channels can be studied with the same concentrations as for $100 \mu \mathrm{m}$ channels, with an obvious loss in optical density. On basis of dilution experiments we anticipate that channels as thin as $20 \mu \mathrm{m}$ should still work well for quantitative transmitted light microscopy. The concentration of Fast Green may still be increased for such studies.

Table 1. UV-vis data, solubility in PBS, maximum recommended concentration, and recommended dye concentration for studying a $100 \mu \mathrm{m}$ high channel. Recommendation takes into account solubility and acceptable optical densities

\begin{tabular}{lcccc}
\hline & $\begin{array}{l}\varepsilon_{\max } / \mathrm{L} \mathrm{mol}^{-1} \\
\mathrm{~cm}^{-1}\end{array}$ & $\lambda_{\max } / \mathrm{nm}$ & $\begin{array}{c}C_{\max } \\
/ \mathrm{mM}\end{array}$ & $\begin{array}{c}C_{100 \mu} \\
/ \mathrm{mM}\end{array}$ \\
\hline Tartrazine $^{(\mathrm{a})}$ & $2.5( \pm 0.2) \times 10^{4}$ & $427.8( \pm 0.6)$ & $4^{(\mathrm{c})}$ & 4 \\
Fluorescein $^{(\mathrm{b})}$ & $7.4( \pm 0.6) \times 10^{4}$ & $490.5( \pm 0.4)$ & $0.4^{(\mathrm{d})}$ & 0.4 \\
Allura Red AC $^{(\mathrm{a})}$ & $2.4( \pm 0.1) \times 10^{4}$ & $497.5( \pm 0.4)$ & $4^{(\mathrm{c})}$ & 4 \\
Amaranth $^{(\mathrm{a})}$ & $2.3( \pm 0.2) \times 10^{4}$ & $521.0( \pm 0.5)$ & $4^{(\mathrm{c})}$ & 4 \\
Erythrosin B $^{(\mathrm{b})}$ & $9.8( \pm 0.2) \times 10^{4}$ & $526.3( \pm 0.1)$ & $0.5^{(\mathrm{e})}$ & 0.5 \\
Rose Bengal $^{(\mathrm{b})}$ & $9.5( \pm 0.5) \times 10^{4}$ & $549.4( \pm 0.2)$ & $0.5^{(\mathrm{e})}$ & 0.5 \\
Fast Green FCF $^{(\mathrm{a})}$ & $1.2( \pm 0.1) \times 10^{5}$ & $623.2( \pm 0.1)$ & $4^{(\mathrm{c})}$ & 1 \\
\hline AuNP-TA, 13 nm $^{(\mathrm{f})}$ & $2.4 \times 10^{8(\mathrm{~g})}$ & 525.5 & n.a. & $0.3 \mu \mathrm{M}$ \\
\hline
\end{tabular}
(a) solution prepared from the sodium salt as received (see text)
(b) solution prepared from dye in its free acid form as received (see text)
(c) no higher concentrations were investigated (see text)
(d) solubility limited
(e) aggregation phenomena may lead to nonlinearities $>0.5 \mathrm{mM}^{23}$
(f) solvent: $1 \mathrm{mM} \mathrm{NaOH}(\mathrm{aq})$
(g) literature value $\mathrm{e}^{32}$

\subsection{Practical considerations}

All solutions (i.e. the dyes in PBS at millimolar concentrations) were stored in the dark at $4^{\circ} \mathrm{C}$ in glass vials or Nalgene flasks. Under these conditions, the dyes solutions are usable in transmitted light micrscopy for several months. Comparison of the UV-visible spectra of aged solutions with freshly prepared solutions showed no significant degradation of the solutions after 3 months for tartrazine, amaranth, allura red or fast green. The latter two were verified to be stable even after 9 months. The spectra of the solutions of the xanthene dyes (Fluorescein, Erythrosin B, Rose Bengal) showed small signs of degradation after 3 months. The degradation manifests itself in UV-visible spectroscopy as a minute but detectable blue shift of the absorption maxima $(\sim 0.5 \mathrm{~nm})$, and a slight decrease of the maximum absorbance 
$(<5 \%)$. This small degradation does not significantly affect the measurements in microfluidic channels, but nevertheless indicates that fresh solutions should be prepared after 3 months.

In contrast to the frequently used fluorescent dye Rhodamine $\mathrm{B}^{33}$ none of the dyes reported here stains PDMS, which means that many experiments involving different dye solutions can be performed on the same PDMS device, without degradation due to irreversible staining of the PDMS channel walls. Rinsing the devices with PBS or pure water rapidly restores the PDMS device to their initial uncoloured state.

\section{Quantitative measurements of dyes in transmitted light microscopy using full-colour imaging}

\subsection{Measurements of optical densities using a colour camera in transmitted light microscopy}

The transmittance of light can be converted into absorbance, and subsequently into concentration values using the well-known Beer-Lambert law. One of the conditions for the Beer-Lambert law to hold is that the light used be monochromatic (or at least that neither the extinction coefficient of the substance nor the probe light intensity vary over the wavelength interval used). Using non-ideal conditions, i.e. using spectroscopic bandwidths that are large compared to the width of the spectral features, leads to the introduction of stray light terms into the Beer-Lambert formula, and to a loss of linearity of the absorbance signal. ${ }^{14}$

Most digital colour cameras divide the visible spectrum into three wide wavelength ranges using Bayer filters, which consist of $2 \times 2$ pixel blocks containing 2 green pixels and 1 red and 1 blue pixel..$^{34}$ Each pixel block gives rise to one colour pixel containing three intensity values for the red, green and blue (RGB) channels. The spectral responses for the red, green and blue channels are similar among CCD cameras. In view of the very large spectral bands used in the Bayer filter for each colour channel, the pixel-wise application of the Beer-Lambert law to digital colour images is expected to suffer from deviations from the ideal linear behaviour. This limitation will depend in particular on the degree of spectral overlap of the dye used with the spectra of the light source (often a tungsten-halogen lamp) and the spectral response of the Bayer filter for the colour channel. Each particular combination of light source, microscope and colour camera will to some extent show a different spectral response, and therefore it is necessary to calibrate the specific response for a dye solution for a given 
microscope, and to determine the concentration range in which the response is linear. Here we will demonstrate the method for one particular microscope set-up. We will also compare the results for that set-up with a different microscope set-up, showing that the small difference in colour responsitivities necessitate calibration and linearity checks for each dyemicroscope combination.

The microfluidic studies of the dye solutions were carried out on a specifically designed microfluidic platform (Figure 2), dubbed 'Péclet-O-Matic', which is used alternatively as a simple flow cell, a T-sensor, an H-filter or a passive mixer. The passive mixer may include an additional staggered herringbone ${ }^{35}$ motif (see Figure SI-6). The channels were fabricated by moulding PDMS against a photolithographically defined SU8 mould. The resulting PDMS slab was bound to microscope slides or cover slips using plasma bonding (see Experimental Section). The focal plane for observation was chosen so as to be half-way the channel height. This results in the channel borders (and other out-of-focus contributions) being slightly blurred, and steep edges in the image profile being smoothed.

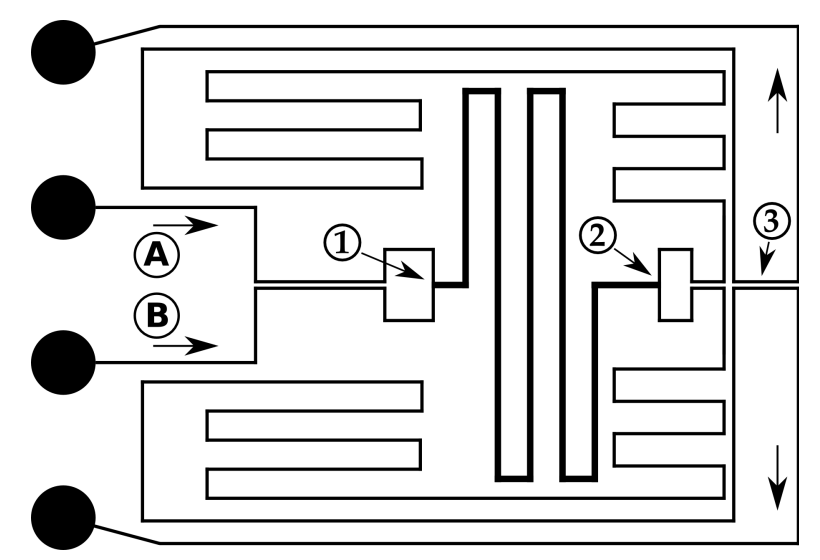

Figure 2. Microfluidic architecture of the 'Péclet-O-Matic'. The straight arrows indicate flow direction. " $A$ " and " $B$ " indicate the two input channels that join at " 1 ", and are split again at "2". Optical measurement of dye concentration in each channel takes place at " 3 ".

The device contains two $100 \mu \mathrm{m}$ wide and $100 \mu \mathrm{m}$ high input channels ("A" and "B", Figure 2) that meet at a $\mathrm{T}$ junction ("1"). Then follows a $54 \mathrm{~mm}$ long, $200 \mu \mathrm{m}$ wide interaction zone, after which the flows are split (point "2", Figure 2). Extra channel length (100 mm each) is then added to homogenize the concentration profile before optical detection at point "3" 
(Figure 2). Concentration of dye at any point in the microfluidic channel may be obtained from transmitted light microscopy through Beer-Lambert law.

Application of Beer-Lambert law requires measurement of incident $\left(I_{0}\right)$ and transmitted $(I)$ light intensities. $I_{0}$ and $I$ can be taken from two separate images (a reference image and the image of the microfluidic channels with dye solutions), but $I_{0}$ values may also be obtained by interpolating a baseline between two transparent regions on the image. The advantage of the latter method is that it is insensitive to changes in lighting conditions and electric gain settings between images. The former method is similar to a flat-field correction in which the image is corrected for inhomogeneous illumination and imaging conditions.

It is essential to optimise light intensity and image acquisition parameters. We found it beneficial to include colour ("daylight") correction filters in the microscope illumination path. Many microscopes have this installed as an option. A BG34 glass filter may be used as well. The effect of this filter is to diminish the red light intensity, thus enhancing the contribution of the blue (and green) channels. The light source should always be operated at the same current setting, since the lamp emission spectrum depends on the temperature of the filament. If necessary, grey neutral density filters can be used to attenuate the light, in order to avoid changing the lamp current. Before beginning actual measurements the light source was left to stabilise at a given current setting (30 minutes, typically). Histograms of the intensity values for each colour channel in the images recorded can be used for checking stable operation.

The camera should be set to output a signal linearly proportional to the light intensity. Any "gamma" correction should be switched off. Importantly, any automatic white balance and background correction, as well as automatic gain adjustments should be switched off as well. The electronic gain for each colour channel needs to be adjusted manually, and should be kept minimal in order to reduce noise. The gain settings can be adjusted to equilibrate the levels of each colour channel (which corresponds to performing a manual white balance). Most scientific and industrial imaging cameras allow for precise parametrization either via the acquisition software of directly on the camera. 
Any dark offset should be set such that in the absence of light on the camera the electric signal (pixel value) is low, but never zero. This is necessary for avoiding the truncation of low-level noise values that would occasionally drop below zero and for which the analog-todigital conversion will simply return zero. A proper dark-corrected image in the absence of light should have noise fluctuating around zero.

For optimising lighting and imaging settings we found it useful to record images of transparent zones of the microfluidic devices and analyse the intensity histograms for each image colour channel. Light intensity and manual gain and dark offset are then regulated such that dark images produce an intensity of $5 \%$ of the maximum value ( 15 in 8 -bit colour values) and transparent, uncoloured zones produce an intensity of $80 \%$ of the maximum value ( 200 in 8-bit values). Signal-to-noise can be significantly improved by averaging ${ }^{\dagger}$ over several images, taken from the same zone using identical illumination and gain settings.

With the image acquisition having been optimised, we can now record images and the process them to obtain three-colour absorbance profiles. For the description of the image processing that follows, each colour channel of the image is considered separately, giving a (monochromatic) matrix $\mathbf{M}^{(\mathrm{R})}, \mathbf{M}^{(\mathrm{G})}$ or $\mathbf{M}^{(\mathrm{B}))}$ for each colour channel. These image matrices are conditioned such that they contain only the part of the image that will contribute to the generation of the absorbance profile. Additionally, the image is oriented such that fluid propagation is oriented along the matrix columns and the absorption profile to be extracted is in the matrix row direction (See the top of Figure 3). The dimensions of $\mathbf{M}^{(\mathbf{R}, \mathbf{G} \text { or B)}}$ are $N_{\text {lines }} \mathbf{X}$ $N_{\text {points, }}$ where $N_{\text {lines }}$ is the number of image lines to be summed and $N_{\text {points }}$ are the number of points in the profile to be extracted.

Both a raw image $\mathbf{M}_{\text {raw }}{ }^{(R, G, B)}$ and a dark image $\mathbf{M}_{\text {dark }}{ }^{(R, G, B)}$ are recorded. The dark image is obtained by blocking the light source, and will be used to correct for the camera dark current. The dark corrected image matrix $\mathrm{M}$ is then obtained (Equation 1).

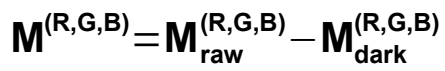

${ }^{+}$This averaging should be done using high bit depth (16-bit, 32-bit) or even floating point digital images 
Additionally it may be possible to record a flat-field image $\mathbf{M}_{\text {bright }}{ }^{(R, G, B)}$ by recording a completely white (and structureless) zone of the device, and use this image to correct (Equation 2) for variations in the illumination and imaging efficiency ('vignetting').

$$
M^{(R, G, B)}=\frac{M_{\text {raw }}^{(R, G, B)}-M_{\text {dark }}^{(R, G, B)}}{M_{\text {white }}^{(R, G)}-M_{\text {dark }}^{(R, G)}}
$$

From the image $\mathbf{M}^{(\mathrm{R}, \mathrm{G}, \mathrm{B})}$ we can extract an intensity profile by creating a row vector whose components are obtained by summing over each of the colums (Equation 3 ). The matrix $\mathbf{1}_{1} \mathrm{x}$ $\mathrm{N}_{\text {lines }}$ is a row matrix of which all of the $N_{\text {lines }}$ elements are 1 (a $1 \times N_{\text {lines }}$ 'ones' matrix). The middle part of Figure 3 shows an example of one of the three colour profiles obtained from the image in the top.

$$
I^{(\mathrm{R}, \mathrm{G}, \mathrm{B})}=1_{1 \times N_{\text {lines }}} \mathrm{M}^{(\mathrm{R}, \mathrm{G}, \mathrm{B})}
$$

A baseline profile $\mathbf{I}_{0}{ }^{(\mathrm{R}, \mathrm{G}, \mathrm{B})}$ is obtained via interpolation by defining the PDMS areas outside the fluidic channels as zones on which the baseline is modeled (e.g. by a linear or a parabolic function). The middle part of Figure 3 shows an interpolated baseline of the sort. In the case of an image that has been flat-field corrected (Equation 2), the baseline will be a constant (ideally equal to 1 ), and interpolation will not be necessary. However, obtaining $\mathbf{I}_{0}$ directly from the image by interpolation is a convenient and robust method and may in many cases be more efficient than separate flat-field corrections. The absorption (or optical density) profiles for each colour channel are obtained by application of Equation 4 .

$$
\mathbf{A}^{(\mathbf{R}, \mathbf{G}, \mathbf{B})}=-{ }^{10} \log \frac{\mathbf{I}_{\text {prof }}^{(\mathbf{R}, \mathbf{G}, \mathbf{B})}}{\mathbf{I}_{\mathbf{0}}^{(\mathbf{R}, \mathbf{G}, \mathbf{B})}}
$$

Row vectors $\mathbf{A}^{(\mathrm{R}, \mathrm{G} \text { or } \mathrm{B})}$ then contain the absorbance (optical density) profiles and have dimensions $\left(1 \times N_{\mathrm{pts}}\right)$. An example of the final set of colour optical density profiles is at the bottom part of Figure 3. Near the microchannel edges the image and the ensuing optical density profile is slightly distorted, due to refraction at the liquid-PDMS interface. This stems from a refractive index mismatch between the aqueous solution chosen and the PDMS. The affected regions were not included in any further analysis. If necessary, the solvent composition may be changed such that its refractive index matches that of the microsystem 
material. ${ }^{1}$ For PDMS, $n \sim 1.43$, this may be achieved using water-glycerol mixtures. This should then be combined with a new calibration of the concentration measurement (see Section 3.2 below).
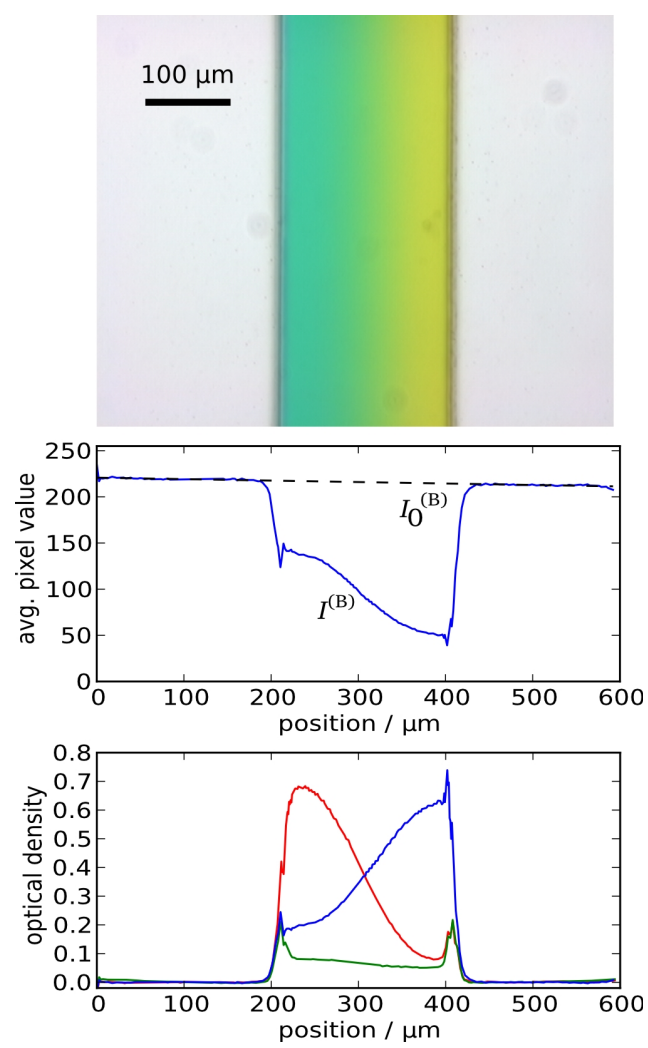

Figure 3. Quantitative transmitted light microscopy of a channel containing interdiffusing laminar flows of Fast Green and Tartrazine in PBS. Top: colour transmitted-light micrograph (10x, NA 0.22 objective) taken in a fluidic channel (just before point "2", Figure 2), and positioned such that the profile to be extracted runs horizontally (along the matrix row direction). The image has the dark signal already subtracted. Middle: intensity profile (I) for the blue channel (solid line) obtained by summing all column elements, baseline $\left(\boldsymbol{I}_{0}\right)$ (dotted line) obtained through linear interpolation. Bottom: optical density (absorbance) profiles for each of the colour channels.

The set of three row vectors containing the colour intensity profiles can be combined into a 3 $\mathrm{x} N_{\text {pts }}$ matrix containing the optical densities with each row containing an intensity profile corresponding to a particular colour. The number of colours - or wavelength bands - may be extended when more wavelength channels are used, such as in multispectral imaging. ${ }^{9}$ The three-colour absorbance profile matrix (which we will refer to as $\mathbf{A}$ ) can be subjected to analytical chemical multicomponent analysis, provided that the individual absorbance 
responses are linearly proportional to the concentration (mathematically 'homogeneous') and additive. These two conditions will be verified experimentally in the two next sections.

\subsection{Linearity: Effective molar absorption coefficients (EMACs)}

The Beer-Lambert law can be cast in the matrix form of Equation 5.

$$
\mathbf{A}=l \mathbf{E C}
$$

(Equation 5)

Matrix A contains the absorbance (optical density) profiles and has dimensions ( $N_{\text {channels }} \mathrm{x}$ $N_{\text {pts, }}$ with $N_{\text {channels }}=3$ for RGB imaging). The optical pathlength is denoted $l$, and is taken to be equal to the channel height. The actual channel height of each device mould was measured using a stylus profilometer $(102 \ldots 110 \mu \mathrm{m})$. Strictly speaking, the assumption that $l$ equals the channel height is only valid for light that traverses the channel perpendicularly. However, a microscope objective collects light over a range of angles, defined by a cone with an aperture 2arcsin(NA), with NA being the numerical aperture of the objective. This leads to effective optical pathlengths larger than the channel height, and may introduce a systematic error for objectives with large NAs. Broadwell et al. found ${ }^{1}$ that for objectives of $10 x$ or less these errors are negligble $(2.3 \%$ for $10 x$, NA 0.3$)$. Therefore, the approximation that optical pathlength $l$ equals the channel height is valid, for the low NA objectives used in this work (NA $0.06 \ldots$ NA 0.30), but higher NA objectives should be avoided, or properly corrected for.

The matrix $\mathbf{C}$ is an $\left(N_{\text {comp }} \times N_{\text {points }}\right)$ matrix which will contain the concentration profiles of each of the components, with $N_{\text {comp }}$ being the number of components and $N_{\text {points }}$ the number of data points representing the concentration profile. In normal UV-visible or IR absorption spectroscopy, matrix $\mathbf{E}$ would contain the molar absorption coefficients ( $\left.N_{\text {channels }} \times N_{\text {comp }}\right)$. Since we are dealing with broad-band, non-monochromatic probe light we prefer to refer to the elements of $\mathbf{E}$ as effective molar absorption coefficients (EMAC).

In this section, we are interested in verifying experimentally a linear relationship between the concentration and the optical density for individual dyes $\left(N_{\text {comp }}=1\right)$. For our RGB images the matrix $\mathrm{E}$ is therefore constructed as in Equation 6.

$$
\mathbf{E}=\left(\begin{array}{l}
E^{E M A C} \\
E_{\text {RED }} \\
E_{\text {MAACEN }} \\
\text { BLUE }
\end{array}\right)
$$


Dilution series were made for each of the dyes investigated by using a staggered herringbone mixer integrated in the interaction zone (between points "1" and "2", Figure 2; see Figure SI-6 for a micrograph), supplying one input with a concentrated solution of dye in PBS, the other with only PBS. The volume fraction of the dye solution is then varied between $0 \%$ and $100 \%$, while keeping the total flow rate constant. The optical density of the solution is measured at the output of the mixer (just before point "2", Figure 2). The concentration accross the microfluidic output channel will be constant, and we take the average optical density accross the channel (reducing $N_{\text {points }}$ to 1 ).

Interestingly, linear behaviour was observed for all dyes in the concentration range tested (typical dilution curves are shown in Figure SI-1, Supporting Info). This is a non trivial experimental result since we are working with non monochromatic detection, under conditions where stray light may be significant. This said, the optical densities used never exceed 1 meaning that at the most, $90 \%$ of the incident light is absorbed, which likely contributes to obtaining linear absorbance-concentration relationships. For both qualitative and quantitative imaging it is beneficial to remain in the concentration range where the solutions absorb at most $90 \ldots 95 \%$ of the light (OD $1 \ldots 1.3$ ) in any of the colour channels. If saturated colours are sought after (in qualitative imaging) there is no need to aim beyond OD 2 , meaning that dye solution concentrations remain in the millimolar range for $100 \mu \mathrm{m}$ channel height.

Table 2 compiles the EMACs determined for the dyes on a particular light source microscope - colour camera combination (see Experimental Section). Surprisingly, certain EMACs are slightly negative (a negative slope of the optical density-concentration plot). This may be attributed to an electronic artefact (negative cross-talk), and/or fluorescence emission. Even for quantitative measurements this is not a problem: the negative EMAC value for a dye should indeed be included as such in the application of Equation 5. 
Table 2. Effective molar absorption coefficients for each colour channel for the principal light source - microscope-camera combination used in this work.

\begin{tabular}{|c|c|c|c|}
\hline & $\begin{array}{l}\mathrm{EMAC}_{\mathrm{RED}} \\
/ \mathrm{mM}^{-1} \mathrm{~mm}^{-1}\end{array}$ & $\begin{array}{l}\mathrm{EMAC}_{\mathrm{GREEN}} \\
/ \mathrm{mM}^{-1} \mathrm{~mm}^{-1}\end{array}$ & $\begin{array}{l}\text { EMAC }_{\text {BLUE }} \\
/ \mathrm{mM}^{-1} \mathrm{~mm}^{-1}\end{array}$ \\
\hline Tartrazine & $-0.02 \pm 0.01$ & $0.12 \pm 0.01$ & $1.4 \pm 0.1$ \\
\hline Fluorescein & $-0.31 \pm 0.13$ & $1.2 \pm 0.1$ & $4.2 \pm 0.2$ \\
\hline Allura Red & $-0.13 \pm 0.01$ & $1.0 \pm 0.1$ & $1.3 \pm 0.1$ \\
\hline Amaranth & $0.05 \pm 0.03$ & $1.9 \pm 0.2$ & $0.9 \pm 0.1$ \\
\hline Erythrosine B & $-0.75 \pm 0.08$ & $3.8 \pm 0.2$ & $1.5 \pm 0.1$ \\
\hline Rose Bengal & $0.01 \pm 0.09$ & $4.5 \pm 0.2$ & $-0.16 \pm 0.03$ \\
\hline Fast Green & $7.0 \pm 0.4$ & $0.52 \pm 0.05$ & $0.29 \pm 0.02$ \\
\hline AuNP-TA $^{(a, b)}$ & $(3.9 \pm 0.8) \times 10^{3}$ & $(14 \pm 3) \times 10^{3}$ & $(10 \pm 2) \times 10^{3}$ \\
\hline
\end{tabular}

(a) $1 \mathrm{mM} \mathrm{NaOH}$ (aq), instead of PBS

(b) uncertainty on actual NP concentration dominates the interval on EMAC values

Since EMACs depend on the spectral overlap between the probe light spectrum, the spectral response of all optics and camera colour channels and the dye, they will depend on the equipment used, and need to be characterised for each particular set-up. Differences are not expected to be enormous, since the spectral response of Bayer filters and silicon-based detectors do not change much between cameras. We tested this by using a second alternative set-up (see Experimental Section). Indeed, the EMACs of a selection of dyes follow the same trend as in Table 2, although their absolute values can deviate by more than $50 \%$ between microscopes (see Supporting Info). We noted that the separation between colour channels was less efficient in the lower cost CMOS camera compared to the CCD camera. In particular the blue channel was less efficient, and in this channel linearity was lost for tartrazine at $>2$ mM.

The instrument-dependence of EMACs contrast with the instrument-independence of conventional molar absorption coefficients determined under near-ideal spectroscopic conditions. Such near-ideal conditions may be created in an optical microscope by using narrow optical band-pass filters, an approach successfully employed for quantifying channel heights and indicator concentrations in microfluidics. ${ }^{1,2}$ The aim of the present work is to establish to what extent unmodified RGB cameras may be used for quantitative concentration measurements, so that colour micrographs can be taken that are visually pleasing and at the 
same time contain useful quantitative data. Moreover, the simultaneous recording of three colour channels provides in principle simultaneous access to the concentrations of three components.

\subsection{Additivity of colour responses}

In order to obtain concentrations from optical densities for dyes with previously measured EMACs, Equation 5 can be inverted (Equation 7).

$$
\mathbf{C}=\frac{1}{l}\left(\mathbf{E}^{-1} \mathbf{A}\right)
$$

For multicomponent analysis $\left(\mathrm{N}_{\text {comp }}>1\right)$ it is important that the colour responses for individual dyes be additive. We tested this condition for several combinations of dyes. Again, a staggered herringbone mixer was used, in combination with a variation of the fractional flow rates of the dye solution at each input, keeping the total absolute flow rate constant. The three-colour optical density row vector that was measured at the output (just before point "3", Figure 2) was converted into a two-component concentration vector using Equation 7 and a $3 \times 2$ E matrix containing the three EMACs for the two components. Figure 4 shows an example of how the concentrations of each of the two components can be calculated from the three optical densities, with the faithful linear gradient proving the additivity of the absorbance response for the two dyes in question.

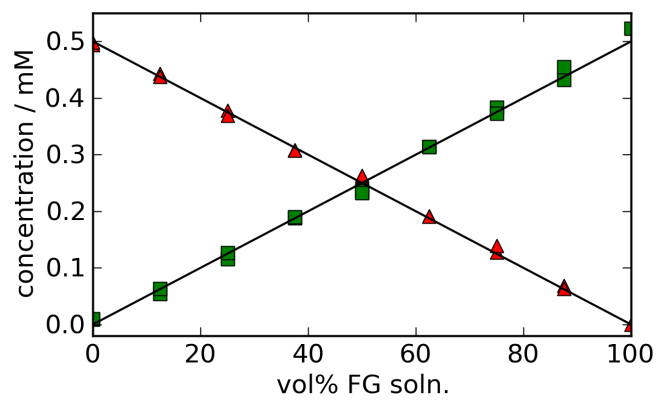

Figure 4. Experimental confirmation for the additivity of optical densities of Rose Bengal and Fast Green. 0.5mM Rose Bengal, 0.5mM Fast Green (both in PBS solution), total flow rate $4 \mu \mathrm{l} \mathrm{min}{ }^{-1}$. The fractional flow rate of the Fast Green solution was increased from 0 to $100 \%$, with the Rose Bengal channel being decreased from 100\% to 0 . The color optical densities were measured at the end of a mixing channel (103 $\mu \mathrm{m}$ high) containing a staggered herringbone motif, and converted into concentrations of the individual components using the inverse of the $3 \times 2$ matrix containing the EMACs for each of the dyes. 
The simultaneous optical measurement of the concentration of each of the two dyes (Fast Green and Rose Bengal) reproduces the concentration gradient generated by varying the relative flow rates of each of the dye solutions. This confirms the additivity of the optical responses of the two dyes. Thus, it is possible to use food dyes for quantitative multicomponent measurements in transmitted light microscopy. The approach can naturally be extended to other dyes, in particular to indicators which exist as a mixture of two species whose relative contribution depends on the concentration of an analyte. Also solutions of gold (and silver) nanoparticles in combination with small dye molecules may be analysed. In addition to a separation according to colour response (EMACs), such samples may also be separated according to diffusion coefficients.

\section{Ratiometric determination of diffusion coefficients using an $\mathrm{H}$ - filter architecture}

Using the dye concentration measurement in transmitted-light microscopy on microfluidic systems, we implement and validate a simplification of diffusion measurements using an $\mathrm{H}$ filter, that is based on the (optical) measurement of the ratio of the concentrations in the channels at the output of the H-filter (point "3", Figure 2).

Near the interface between two (infinitely wide) laminar flows, one of which initially contains all the analyte at concentration $C_{0}$, the other only solvent, the temporal evolution of the analyte concentration profile is usually calculated using Equation $8 .{ }^{36,37}$

$$
C(x, t)=\frac{1}{2} C_{0}\left(1-\operatorname{erf}\left(\frac{x}{2 \sqrt{D t}}\right)\right) \text { with } \operatorname{erf}(z)=\frac{2}{\sqrt{\pi}} \int_{0}^{z} \exp \left(-v^{2}\right) d v
$$

In Equation 8, $x$ represents the position across the channel, with $\mathrm{x}=0$ being the position of the flow interface. Constant flow converts time $t$ into a spatial coordinate along the channel. In the microfluidic T-sensor ${ }^{15-18}$ architecture, diffusion coefficients are extracted by fitting Equation 8 to steady-state fluorescence microscopy images of the T-junction. ${ }^{5,36}$ This procedure is limited to fluorescent entities, or to entities that can be detected using fluorescent probes (such as ${ }^{38} \mathrm{H}^{+}$and ${ }^{39} \mathrm{Ca}^{2+}$ ).

We propose a modified microfluidic measurement of diffusion coefficients based on the measurement of the concentration ratio between two laminar flows at the output of a H-filter. 
The two laminar flows, one initially empty and one containing the substance under analysis, meet at a T-junction are allowed to interact in a long channel, after which they are separated. Subsequently, the analyte concentration ratio of the two outputs is measured. In the present work, the concentration measurement will be done using transmitted light microscopy, using the methodology described above, but may be replaced by other means of concentration measurement (electrochemistry for example). Compared to fluorescence imaging, this method greatly increases the range of species whose diffusion coefficient can be determined, for example to the nonfluorescent food dyes that we aim to characterise in view of their application in quantitative microfluidic analysis.

Equation 8 is for the evolution of a front between two infinitely broad flows, one of which initially contains all the analyte, the other only solvent. Defining the $\mathrm{H}$ filter as a microfluidic channel (of limited width $w$, height $h$, length $L$ ) where two laminar flows (A and B) interact, an analytic expression for the temporal evolution of the concentration profile across a channel of limited width can be obtained using a Fourier approach. Such an approach has for example been used in the systematic design of gradient generators, ${ }^{40}$ and has indeed been suggested for describing diffusion in microchannels. ${ }^{41}$ In the case of the $\mathrm{H}$ filter architecture used here we find

$$
C(x, t)=\frac{C_{0}}{2}+\sum_{k=0}^{\infty}(-1)^{k} \frac{2 C_{0}}{\pi(2 \mathrm{k}+1)} \exp \left(\frac{-D \pi^{2}(2 \mathrm{k}+1)^{2}}{w^{2}} t\right) \cos \left(\frac{\pi(2 \mathrm{k}+1)}{w} x\right)
$$

At the input of the H-filter flow A contains a solution of the analyte (dye) at a concentration $C_{0}$. Flow B contains only the solvent (buffer). At the output of the H-filter both flows are separated (e.g. point "3" in Figure 2). The concentrations of the analyte in each of the separated flows at the output are denoted $C_{A}$ and $C_{B}$ for flows $A$ and $B$, respectively (Equation 10).

$$
C_{\mathrm{A}}=\frac{2}{w} \int_{0}^{w / 2} C(x, t) d x \text { and } C_{\mathrm{B}}=\frac{2}{w} \int_{w / 2}^{w} C(x, t) d x
$$

For the determination of diffusion coefficients using the ratiometric $\mathrm{H}$ filter method, a natural way of expressing the experimental data may be plotting the ratio of $C_{B}$ and $C_{A}$ (equal to the ratio of the absorbances, see below) as a function of flow rate. Using Equations 2 and 3, this ratio can be expressed as Equation 11. 


$$
\frac{C_{\mathrm{B}}}{C_{\mathrm{A}}}=\frac{1-\xi}{1+\xi}
$$

with

$$
\xi=\sum_{i=0}^{\infty} c_{i} \exp \left(-k_{i} t\right)
$$

and $\quad c_{i}=\frac{8}{\pi^{2}(2 \mathrm{i}+1)^{2}}$ and $k_{i}=\frac{D \pi^{2}(2 \mathrm{i}+1)^{2}}{w^{2}}$

The $\xi$ function describes the extent of mixing (i.e. equal distribution of the analyte between flows A and B), and is related to the observed concentration ratio according to Equation 13.

$$
\xi=\frac{C_{\mathrm{A}}-C_{\mathrm{B}}}{C_{\mathrm{A}}+C_{\mathrm{B}}}
$$

These expressions describe the evolution of the (one-dimensional) concentration ratio in time. Adding steady flow in a second dimension enables us to express $t$ (which can be assimilated to the average residence time of a molecule in the interaction zone) in terms of the channel dimensions $(h, w$, and $L)$ and the flow velocity $U_{0}$ or the flow rate $V_{\text {flow }}$.

$$
U_{0}=\frac{V_{\text {flow }}}{h w} \text { and } t=\frac{L}{U_{0}}
$$

The series in Equation 9 and 12 converge quite quickly for large $t$ (slow flow rates), and one or two terms are generally sufficient to describe the concentration ratio as a function of flow rate (see Supporting Info, Figure SI-2, for the convergence behaviour of Equation 12). When applying Equation 14 to Equation 12, the Péclet number $\left(P e=U_{0} w / D\right)$ appears naturally.

For solutions containing only a single detectable component we can express the concentration ratio of analyte in flows $\mathrm{A}$ and $\mathrm{B}$ as the ratio of the measured absorbances $\left(\mathrm{OD}_{\mathrm{A}}\right.$ and $\left.\mathrm{OD}_{\mathrm{B}}\right)$, applying Beer-Lambert law. In practice, these absorbance values are obtained by averaging the values in each channel extracted from absorbance profiles obtained at point "3" (Figure 2) using the method described in Section 3.1. The concentration ratios then become optical density ratios, as per Equation 15.

$$
\frac{C_{\mathrm{B}}}{C_{\mathrm{A}}}=\frac{\mathrm{OD}_{\mathrm{B}}}{\mathrm{OD}_{\mathrm{A}}} \text { and } \frac{C_{\mathrm{A}}-C_{\mathrm{B}}}{C_{\mathrm{A}}+C_{\mathrm{B}}}=\frac{\mathrm{OD}_{\mathrm{A}}-\mathrm{OD}_{\mathrm{B}}}{\mathrm{OD}_{\mathrm{A}}+\mathrm{OD}_{\mathrm{B}}}=\xi
$$


At sufficiently slow flow rates we can use only the first term of the series in Equation 12 and linearize Equation 13. This gives Equation 16.

$$
\ln \frac{\mathrm{OD}_{\mathrm{A}}-\mathrm{OD}_{\mathrm{B}}}{\mathrm{OD}_{\mathrm{A}}+\mathrm{OD}_{\mathrm{B}}}=-D \tau+\ln \frac{8}{\pi^{2}}
$$

with $\quad \tau=\frac{\pi^{2} L}{U_{0} w^{2}}=\frac{\pi^{2} L h}{V_{\text {flow }} w}$.

In Equation 16 the only experimentally unknown parameter is the diffusion coefficient $D$ which is the slope of the line obtained by tracing $\ln (\xi)$ as a function of $\tau$. It is interesting to note that $\mathrm{OD}_{\mathrm{A}}$ and $\mathrm{OD}_{\mathrm{B}}$ may be replaced by any other (processed) signal that is linearly proportional to the concentration of the analyte, such as values from amperometry or conductivity. All expressions are in SI units. A total flow rate of $1 \mu 1 \mathrm{~min}^{-1}$ amounts to $V_{\text {flow }}=$ $1.667 \times 10^{-11} \mathrm{~m}^{3} \mathrm{~s}^{-1}$, for a $200 \mu \mathrm{m}$ wide, $100 \mu \mathrm{m}$ high channel.

Figure 5 exemplifies the experimental validity of the method for Allura Red in PBS, and 13 nm diameter AuTA particles in $1 \mathrm{mM}$ aqueous $\mathrm{NaOH}$. The optical densities were taken both from the green and the blue camera channels. The ratios of the optical densities (i.e. the concentrations) of the two H-filter outputs indeed follow the theoretical curve corresponding to diffusion coefficient $D=3.4 \times 10^{-10} \mathrm{~m}^{2} \mathrm{~s}^{-1}$ and $D=0.37 \times 10^{-10} \mathrm{~m}^{2} \mathrm{~s}^{-1}$ for Allura Red and AuTA particles, respectively. These correspond to a Stokes-Einstein hydrodynamic radius of $0.7 \mathrm{~nm}$ for Allura Red and $6.5 \mathrm{~nm}$ for AuTA, in good agreement with the expected radii of the objects. 

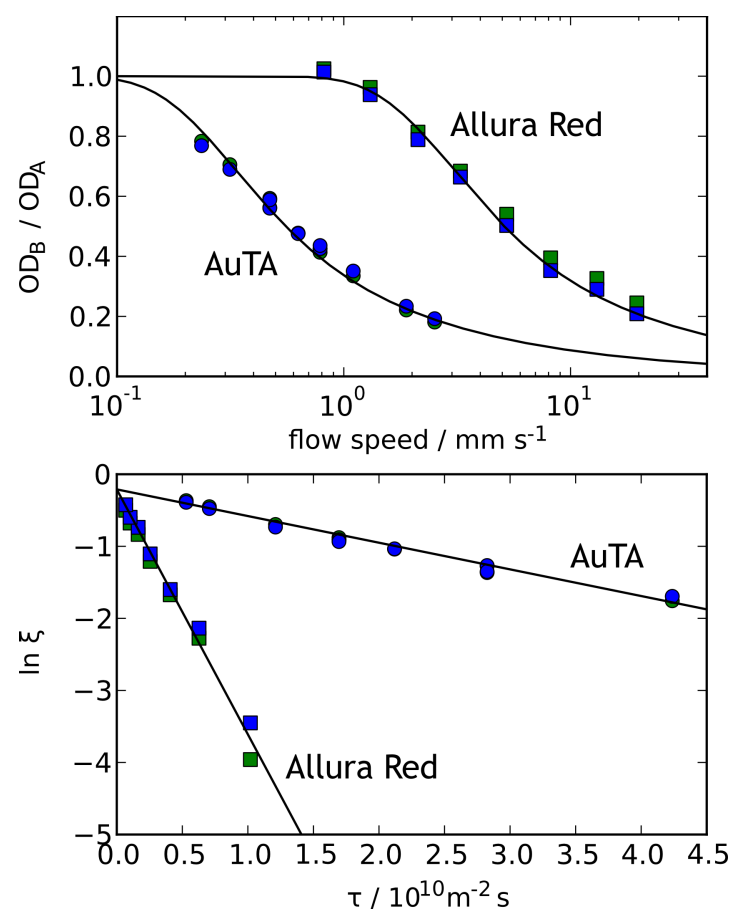

Figure 5. Ratiometric determinations of the diffusion coefficient of Allura Red AC in PBS (squares) and $13 \mathrm{~nm}$ diameter AuTA nanoparticles (circles). The channel width is $200 \mu \mathrm{m}$, height $103 \mu \mathrm{m}$, interaction length $54 \mathrm{~mm}$. Top: Ratio of the measured absorbances of channel $B$ and $A$ as a function of flow velocity. The solid lines are the theoretical curves for $D=3.4 \times 10^{-10} \mathrm{~m}^{2} \mathrm{~s}^{-1}(A R)$ and $D=0.37$ $\times 10^{-10} \mathrm{~m}^{2} \mathrm{~s}^{-1}$ (AuTA). Bottom: The same data plotted in the linearised form of Equation 16, again with $D=3.4 \times 10^{-10} \mathrm{~m}^{2} \mathrm{~s}^{-1}$ for Allura Red, and $\mathrm{D}=3.7 \times 10^{-11} \mathrm{~m}^{2} \mathrm{~s}^{-1}$ for $13 \mathrm{~nm}$ diameter AuTA nanoparticles.

The linearised form of the analysis (Equation 16, bottom of Figure 5) provides a straightforward way of analysing the data, plotting $\ln \left(\left(C_{A}-C_{B}\right) /\left(C_{A}+C_{B}\right)\right)$ as a function of $\tau$, which is inversely proportional to the flow rate. This approach works best at low flow rates (longer mixing times) where $\ln \xi<-0.4\left(C_{B} / C_{A}>0.2\right)$. An analysis of the truncation behaviour of the Fourier series is given in the Supporting Info.

Results for all dyes studied are compiled in Table 3. The reported diffusion coefficients are the averages over several measurements on different devices. Not surprisingly, all dye diffusion coefficients are within the same order of magnitude, since all dye molecules have similar dimensions. The value found for fluorescein agrees with values reported in the literature. A separate measurement of fluorescein diffusion was carried out in the Péclet-OMatic using a lower concentration of fluorescein $\left(10^{-4} \mathrm{M}\right)$ and epifluorescence detection, for comparison. In this case, the concentration in each of the channels is given by the integrated 
fluorescence intensity over the channel (instead of the optical density). This fluorescence measurement yielded $\mathrm{D}=6 \times 10^{-10} \mathrm{~m}^{2} \mathrm{~s}^{-1}$, in agreement with the measurements in transmitted-light microscopy.

Table 3. Diffusion coefficients measured using the ratiometric microfluidic $H$-filter method, $D$, and literature or theoretical values $\left(D_{\text {litt }}, D_{\text {theo }}\right.$ ). Reported intervals are the $95 \%$ confidence interval of $D$ measured on several different devices. Temperature was $24( \pm 2)^{\circ} \mathrm{C}$

\begin{tabular}{lcc}
\hline & $\begin{array}{c}D \\
\end{array}$ & $\begin{array}{c}D_{\text {litt }} \text { or } D_{\text {theo }} \\
/ 10^{-10} \mathrm{~m}^{2} \mathrm{~s}^{-1}\end{array}$ \\
\hline Tartrazine & $4.9 \pm 0.8$ & \\
Fluorescein & $6.5 \pm 0.5$ & $6.4^{(\mathrm{a})}, 7.0^{(\mathrm{b})}$ \\
Allura Red & $3.6 \pm 0.4$ & \\
Rose Bengal & $4.8 \pm 0.9$ & \\
Fast Green & $3.9 \pm 0.7$ & \\
AuNP-TA & $0.37 \pm 0.06$ & $0.38^{(\mathrm{c})}$ \\
(13 nm diam.) & & \\
\hline
\end{tabular}

(a) Ref. 41

(b) Ref. 42

(c) Calculated using the Stokes-Einstein relation for a hydrodynamic radius of $6.5 \mathrm{~nm}$.

The diffusion of $13 \mathrm{~nm}$ diameter gold nanoparticles, coated with disulfide ligands, was also studied. The diffusion coefficient $\left(3.7 \times 10^{-11} \mathrm{~m}^{2} \mathrm{~s}^{-1}\right)$ shows good agreement with the coefficient predicted by the Stokes-Einstein equation for spherical particles with a diameter of $13 \mathrm{~nm}$. In addition to the ratiometric diffusion measurement (Equations 11 and 16), we also investigated the evolution of the gold nanoparticle concentration profile at the end of the interaction channel (just before point "2", Figure 2) as a function of flow rate (see Supporting Info, Figures SI-4 and SI-5). The concentration profiles are well described by Equation 9. These results suggest that this type of diffusion measurement may be a useful way for the characterisation of functionalised nanoparticles preparations, especially for the detection of the presence of small molecules, such as desorbed free ligands. ${ }^{43}$

The microfluidic method for diffusion measurement presented here works remarkably well, in spite of its simplicity. It seems to be robust, even at low flow rates using syringe pumps. Small mechanical fluctuations in the syringe pump rate that are visible at the input (point "1", Figure 2) are not observed at the output, and at point "2" a very steady profile is observed, 
that reliably changes as a function of overall flow rate. Since measurement is done at the end of the channel (at relatively long mixing times) complicating factors are likely smoothed out, e.g. with Taylor-Aris dispersion smoothing out the Poiseuille flow profile. Comparison of the "Fourier-based" analysis used in this work and the "erf-based" (Equation 8) analysis shows that the latter works mainly at short mixing times (i.e. at the input of the H-filter/T-sensor), and the former is very effective at moderate to long mixing times (see Supporting Info, Figure SI-3).

Perhaps the fluidic implementation may be improved using flow focussing techniques for reducing wall effects. ${ }^{6}$ However, the current implementation using ratiometric measurement of overall concentrations may be much less sensitive to wall effects as the overall response is averaged out, and the long flow length favorises volume effects over wall effects.

\section{Multicomponent analysis: Characterisation of a passive mixer and a gradient generator}

As an illustration of the multicomponent concentration mapping capability of colour transmitted light microscopy we studied two typical microlfluidic systems: a passive mixer based on the staggered herringbone motif ${ }^{35}$ and a gradient generator. ${ }^{21,44,45}$

A panoramic microscopy image of the staggered herringbone mixer (SHM) was constructed from individual images taken at different positions (see Supporting Info, Figure SI-6). The mixer was operated mixing $0.9 \mathrm{mM}$ fast green and $4.6 \mathrm{mM}$ tartrazine ('blue + yellow = green'), both in PBS. From the individual images we extracted the concentration profiles of each dye before mixing, and after 2, 4, 6, 8 and 10 SHM cycles (Figure 6). Our measurements show that after 10 mixing cycles the concentration profiles of both dyes are completely flat. 

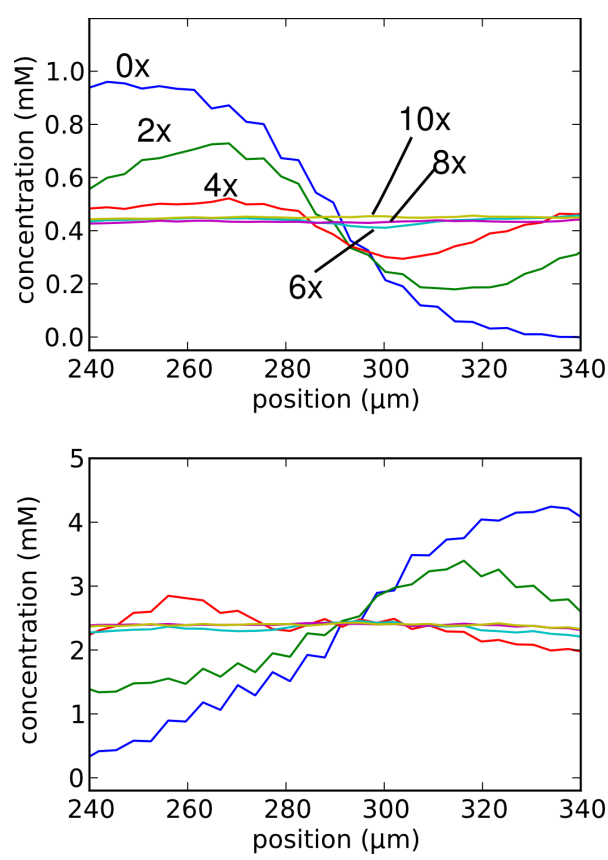

Figure 6. Evolution of the central part of the concentration profiles of Fast Green (top) and Tartrazine (bottom) in a staggered herringbone mixing device (see also Supporting Info for an image of the device) monitored using full-colour transmitted light microscopy and multicomponent analysis. Profiles are shown before (" $\left.0 x^{\prime \prime}\right)$ and after 2, 4, 6, 8, and 10 mixing cycles. Total flow rate in $100 \mu \mathrm{m}$ high, $200 \mu \mathrm{m}$ wide mixing channel: $14 \mu \mathrm{l} \mathrm{min}^{-1}$.

It is interesting to compare the transmitted-light profiles (Figure 6), which are optical densities that are averaged in the direction of light propagation (the " $z$ " direction, from channel top to channel bottom), to (single component) confocal microscopy images previously taken on an SHM that resolve this vertical concentration profile. We then see that the transmitted-light profiles indeed follow (at least semi-quantitatively) the averages along the vertical axis of these confocal images.

In addition to the passive chaotic mixer, we also studied a chemical gradient generator under development for cell studies. Cell toxicology assays are a common application for microfluidic gradient generators. ${ }^{44,45}$ They can also be used as a microfluidic component in the development of chemical micro-reactors for analytical or biomedical applications. Based on well-controlled successive dilutions of initial stock solutions in a large microarray, gradients are generated with the use of multiple T-channels. ${ }^{37}$ Several channels are combined in order to obtain the desired concentration gradient. ${ }^{40}$ 
The device shown in Figure 7 is a simple pyramidal architecture designed for achieving a well-controlled gradient of two stock solutions, distributed over 9 perfusion chambers for cell studies. The device has been designed with a channel height of $75 \mu \mathrm{m}$. Each T-channel has a length of mixing of $5 \mathrm{~mm}$ and a width of $50 \mu \mathrm{m}$. The panoramic image is composed of 31 individual microscope images, each of which has been flat-field corrected (Equation 2).

With the homogenised illumination it is now straightforward to extract concentration profiles. Two of such profiles are shown in Figure 7. Profiles "A" show the profiles at an intermediate stage, where four gradient steps have been generated. Interestingly, as is particularly clear from the encircled part of the profiles, the mixing of the input streams is not entirely complete at the flow rate used. As a result, the middle chamber at the output (profiles "B") does not have exactly a 50:50 mixture of the inital solutions. This is not immediately clear from the overall image, and is only revealed after quantitative determination of the two-component concentration profiles. The device should be operated at a lower flow rate in order to achieve a perfect gradient over the cell perfusion chambers.
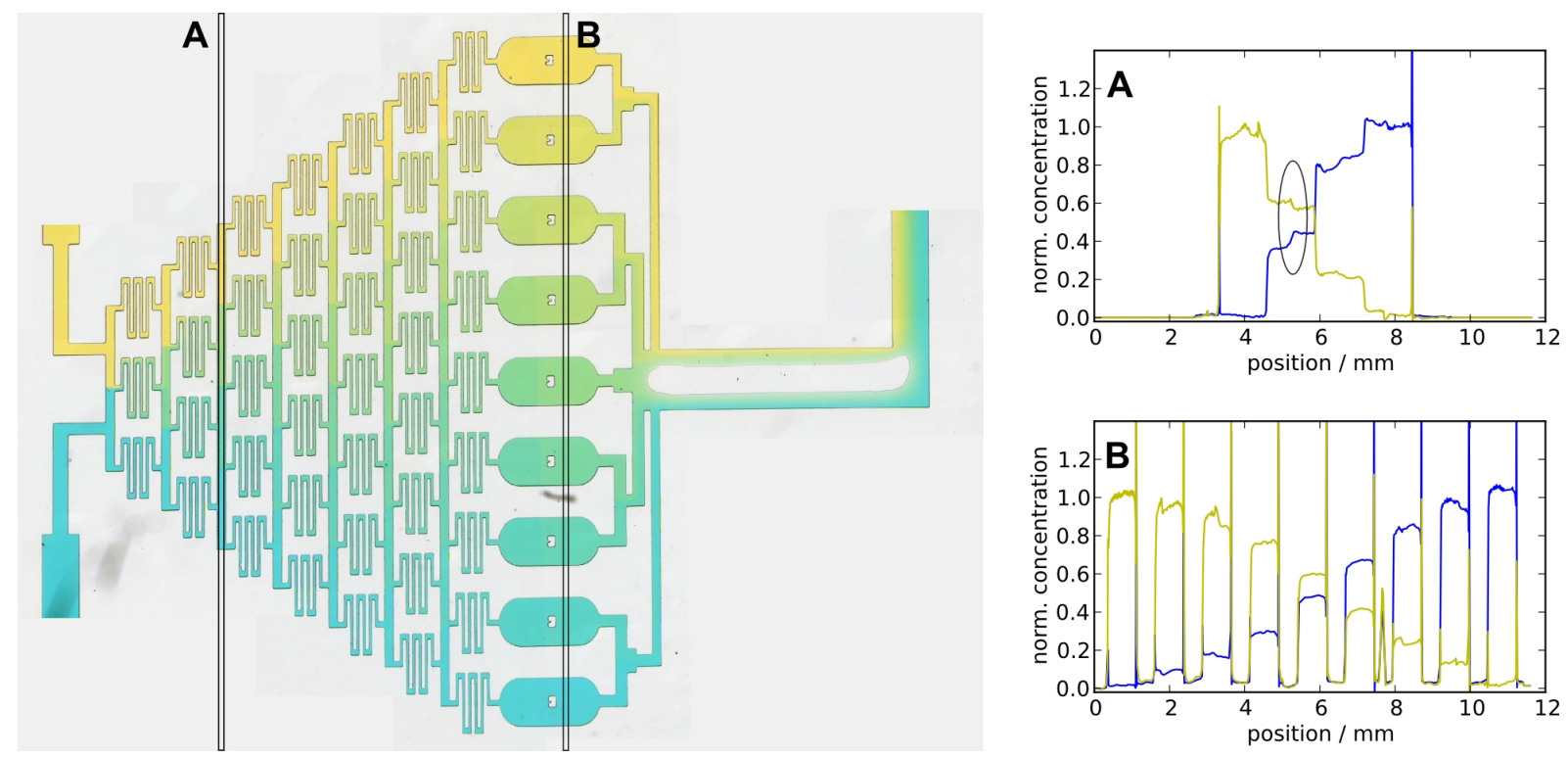

Figure 7. Left: Panoramic picture (multiple images taken using a 2.5x NA 0.07 objective) of a gradient generator (channel height $75 \mu \mathrm{m}$ ) that generates a gradient distributed over 9 perfusion chambers. The input solutions are Tartrazine/PBS and Fast Green/PBS. Right: normalized concentration profiles for Tartrazine (yellow) and Fast Green (blue) determined using multicomponent analysis of the colour optical density profiles. Total flow rate $4 \mu \mathrm{l} \mathrm{min}{ }^{-1}$, which is slightly too high to ensure complete mixing leading to small deviations from ideality, illustrated by the encircled zone of Profiles " $A$ ". 


\section{Conclusion}

The possibility of implementing quantitative multicomponent concentration measurements in microfluidic systems using transmitted light microscopy and full colour imaging has been demonstrated, in spite of the non-monochromatic nature of the Bayer colour filters used in (digital) cameras, which was expected to complicate application of Beer-Lambert law. The methodology presented in this work will help more thorough characterisation of microfluidic architectures with relatively simple equipment. Additionally, it may help implementation of simple detection schemes in (bio)microsystems, when used with coloured indicators ( $\mathrm{pH}$, metals, redox, proteins...). A set of coloured solutions of well-defined composition for microfluidics is proposed, with the trio Fast Green-Amaranth-Tartrazine (cyan-magenta-yellow) being well-suited for use with RGB (red-green-blue) cameras. The methodology may be extended to other dye systems, such as acid-base or metal indicators (and light-absorbing nanoparticles, as demonstrated).

The implementation of quantitative dye concentration measurements using a colour camera enabled us to develop and validate a simplified ratiometric method for the determination of diffusion coefficients using an $\mathrm{H}$-filter architecture. This ratiometric method simplifies and greatly extends the scope of diffusion measurements in microfluidic systems, that can be further extended to include non-optical (e.g. electroanalytical ${ }^{46}$ ) detection methods.

\section{Acknowledgments}

Financial support from ERANET-NanoSciences (project MOLIMEN) and the Agence Nationale de la Recherche (program JCJC2010, project COMONSENS) are gratefully acknowledged.

\section{Experimental section}

\section{Spectroscopy and microscopy}

Spectroscopic and digital imaging data were analysed using home-written software based on the Scipy (0.7.0), Numpy (1.3.0) and Matplotlib (0.99.1.1) libraries for the Python programming language (version 2.6.5), on Windows XP, Mac OS X or GNU/Linux systems. 
$\mathrm{UV} /$ vis absorption spectrometric measurements were performed using an optical fiber-based system (OceanOptics, USA) incorporating a USB4000-VIS-NIR CCD spectrometer and an LS1 tungsten-halogen light source equipped with a BG34 colour correction filter. The data reported are based on the dilution series of at least three independently prepared solutions. The maximum values of the absorption spectra, and their spectral position, were obtained from fitting a parabola through the experimental spectra in a small window $(+/-5 \mathrm{~nm})$ centered around the numerical maximum (which is not necessarily the maximum of the absorption band due to noise).

Transmitted light microscopy was performed on a Leica DMIRB inverted microscope, with a tungsten-halogen light source with "daylight" (DLF) correction filter and a Hitachi KP-D50 CCD colour camera with Pinnacle frame grabber. For measurement of concentration profiles inside a single fluidic channel or concentration ratios between two parallel channel channels, a 10x (NA 0.22) CPlan objective was used. For the panoramic images, a 2.5x (NA 0.07) NPlan objective was employed. For comparison, several experiments were repeated on a Olympus IX71 microscope with a low-cost Thorlabs DCC-1645-C CMOS colour camera, with either a 10x (NA 0.30) CPlan-Fl objective, or a 2x (NA 0.06) PlanN objective.

\section{Microfabrication and device operation}

Microfluidic channels were moulded into polydimethylsiloxane (PDMS, General Electric RTV615) using SU-8 (Microchem, SU8-2100) moulds defined on 2" silicon wafers by standard photolithographic processes. After cross-linking $\left(2 \mathrm{~h} @ 75^{\circ} \mathrm{C}\right)$, the PDMS slab was attached to cleaned microscope glass slides $(25 \mathrm{~mm} \times 75 \mathrm{~mm})$ using low pressure air plasma bonding (Harrick Plasma Cleaner PDC-002, 1 minute). Cleaning of the glass slides involved rubbing with isopropanol $(\mathrm{iPrOH})$ and cleanroom wipes followed by sonication in $1 \%$ Hellmanex II (Hellma) at $35^{\circ} \mathrm{C}$, thorough rinsing with ultrapure water, followed by $\mathrm{iPrOH}$, drying in a nitrogen jet and subsequently in a stove at $70^{\circ} \mathrm{C}$, ending with 15 minutes in the plasma cleaner.

Microfluidic systems were operated with Aladdin (WPI Europe, Hertfordshire, UK) or Harvard (Harvard Apparatus, Holliston MA, USA) syringe pumps and $250 \mu 1 . . .1000 \mu$ glass precision syringes (SGE Europe, Milton Keynes, UK). The fluids were supplied to the devices 
using ETFE Tefzel tubing (Cluzeau, Sainte-Foy-La-Grande, France) containing in-line filters ( $2 \mu \mathrm{m}$ pore diameter, Cluzeau).

\section{Gold nanoparticles}

Thiooctic acid-capped gold nanoparticles of $13 \mathrm{~nm}$ diameter (AuNP-TA) were prepared by adding a solution of thiooctic acid to a solution of excess citrate-stabilised gold nanoparticles. The excess citrate-stabilised gold sol was prepared according to the well-known FrensTurkevich method ${ }^{47,48}$ following the recipe in Ref. 49 . The citrate gold sol was found to have a concentration of $4.2 \times 10^{-9} \mathrm{M}$ in gold nanoparticles, using UV-visible spectroscopy $\left(\varepsilon_{\text {part }}=2.4 \mathrm{x}\right.$ $10^{8} \mathrm{M}^{-1} \mathrm{~cm}^{-1}$ for $13 \mathrm{~nm}$ diameter citrate AuNP). ${ }^{32}$

An aqueous solution containing 2.4 mM NaOH and 2.4 mM DL-thiooctic acid was prepared, and $2.5 \mathrm{ml}$ of this solution was added dropwise to $150 \mathrm{ml}$ of a vigourously stirred gold sol. After stirring for another 30 minutes, the solution was stored in the dark overnight. The solution was concentrated by several cycles of centrifugation $(9700 \times g, 10000 \mathrm{rpm}, 10 \mathrm{~min}$., Hettich Mikro 220r), removal of supernatant (90\% of the volume) followed by replacement of the removed supernatant by additional nanoparticle solution. The concentrate had a final particle concentration of $7.3 \times 10^{-7} \mathrm{M}$. The concentrate was then further purified by diluting it 10 times using $1 \mathrm{mM}$ aqeous $\mathrm{NaOH}$, followed by centrifugation $(9700 \times g$, $10 \mathrm{~min}$.$) , and$ removal of supernatant (90\% of the volume). The pellet was finally diluted 2.5 times using 1 $\mathrm{mM}$ aqueous $\mathrm{NaOH}$, to give a nanoparticle solution at $2.9 \times 10^{-7} \mathrm{M}$. Before disposal, spent nanoparticle solutions were exposed to gold etchant (Transene Gold Etchant TFA, which converts the nanoparticles into gold ions, and thus removes the nanoparticulate nature of the waste). ${ }^{49}$

\section{References}

1 I. Broadwell, P. D. I. Fletcher, S. J. Haswell, T. McCreedy and X. L. Zhang, Quantitative 3-dimensional profiling of channel networks within transparent 'lab-on-a-chip' microreactors using a digital imaging method, Lab Chip, 2001, 1, 66-71.

2 P. D. I. Fletcher, S. J. Haswell and X. L. Zhang, Electrokinetic control of a chemical reaction in a lab-on-a-chip micro-reactor: measurement and quantitative modelling, Lab Chip, 2002, 2, 102-112.

3 D. C. Leslie, B. A. Melnikoff, D. J. Marchiarullo, D. R. Cash, J. P. Ferrance and J. P. Landers, A simple method for the evaluation of microfluidic architecture using flow quantitation via a multiplexed fluidic resistance measurement, Lab Chip, 2010, 10, 1960-1966.

4 C. Hsu and A. Folch, Spatio-temporally-complex concentration profiles using a tunable chaotic micromixer, Appl. Phys. Lett., 2006, 89, 144102. 
5 A. E. Kamholz, E. A. Schilling and P. Yager, Optical measurement of transverse molecular diffusion in a microchannel, Biophys. J., 2001, 80, 1967-72.

6 M. S. Munson, K. R. Hawkins, M. S. Hasenbank and P. Yager, Diffusion based analysis in a sheath flow microchannel: the sheath flow T-sensor, Lab Chip, 2005, 5, 856-62.

7 G. M. Walker, N. Monteiro-Riviere, J. Rouse and A. T. O'Neill, A linear dilution microfluidic device for cytotoxicity assays, Lab Chip, 2007, 7, 226.

8 J. Q. Wu and J. Pawliszyn, Dual detection for capillary isolelectric focusing with refractive index gradient and absorption imaging detectors, Anal. Chem., 1994, 66, 867-873.

9 C. Rothmann, A. M. Cohen and Z. Malik, Chromatin condensation in erythropoiesis resolved by multipixel spectral imaging: Differentiation versus apoptosis, J. Histochem. Cytochem., 1997, 45, 1097-1108.

10 J. Thigpen, F. A. Merchant and S. K. Shah, Photometric calibration for quantitative spectral microscopy under transmitted illumination, J. Microscopy, 2010, 239, 200-214.

11 B. P. Binks, Z. G. Cui and P. D. I. Fletcher, Optical microscope absorbance imaging of carbon black nanoparticle films at solid and liquid surfaces, Langmuir, 2006, 22, 1664-1670.

12 T. A. Everett and D. A. Higgins, Electrostatic self-assembly of ordered perylene-diimide/polyelectrolyte nanofibers in fluidic devices: from nematic domains to macroscopic alignment, Langmuir, 2009, 25, 1304513051.

13 N. Kakuta, Y. Fukuhara, K. Kondo, H. Arimoto and Y. Yamada, Temperature imaging of water in a microchannel using thermal sensitivity of near-infrared absorption, Lab Chip, 2011, DOI: 10.1039/c1lc20261h.

14 G. C. Y. Chan and W. T. Chan, Beer's law measurements using non-monochromatic light sources - a computer simulation, J. Chem. Ed., 2001, 78, 1285-1288.

15 A. E. Kamholz, B. H. Weigl, B. A. Finlayson and P. Yager, Quantitative analysis of molecular interaction in a microfluidic channel: the T-sensor, Anal. Chem., 1999, 71, 5340-7.

16 B. H. Weigl and P. Yager, Microfluidics - Microfluidic diffusion-based separation and detection, Science, 1999, 283, 346-347.

17 J. P. Brody and P. Yager, Diffusion-based extraction in a microfabricated device, Sens. Actuators A, 1997, 58, 1318.

18 T. M. Squires and S. R. Quake, Microfluidics: fluid physics at the nanoliter scale, Rev. Mod. Phys., 2005, 77, 9771026.

19 J. L. Osborn, B. Lutz, E. Fu, P. Kauffman, D. Y. Stevens and P. Yager, Microfluidics without pumps: reinventing the T-sensor and H-filter in paper networks, Lab Chip, 2010, 10, 2659.

20 A. Persat, R. D. Chambers and J. G. Santiago, Basic principles of electrolyte chemistry for microfluidic electrokinetics. Part I: Acid-base equilibria and pH buffers, Lab Chip, 2009, 9, 2437-53.

21 G. A. Cooksey, C. G. Sip and A. Folch, A multi-purpose microfluidic perfusion system with combinatorial choice of inputs, mixtures, gradient patterns, and flow rates, Lab Chip, 2009, 9, 417.

22 C. Neils, Z. Tyree, B. Finlayson and A. Folch, Combinatorial mixing of microfluidic streams, Lab Chip, 2004, 4, 342.

23 O. Valdes-Aguilera and D. C. Neckers, Aggregation phenomena in xanthene dyes, Acc. Chem. Res., 1989, 22, 171-177.

24 C. Grotzinger, D. Burget, P. Jacques and J. P. Fouassier, Visible light induced photopolymerization: speeding up the rate of polymerization by using co-initiators in dye/amine photoinitiating systems, Polymer, 2003, 44, 3671-3677.

25 P. Gottschalk, J. Paczkowski and D. C. Neckers, Factors influencing the quantum yields for rose bengal formation of singlet oxygen, J. Photochem., 1986, 35, 277-281.

26 S. Y. Lin, Y. T. Tsai, C. C. Chen, C. M. Lin and C. H. Chen, Two-step functionalization of neutral and positively charged thiols onto citrate-stabilized Au nanoparticles, J. Phys. Chem. B, 2004, 108, 2134-2139.

27 P. K. Jain, X. H. Huang, I. H. El-Sayed and M. A. El-Sayed, Noble metals on the nanoscale: optical and photothermal properties and some applications in imaging, sensing, biology, and medicine, Acc. Chem. Res., 2008, 41, 1578-1586. 
28 J. Yguerabide and E. E. Yguerabide, Light-scattering submicroscopic particles as highly fluorescent analogs and their use as tracer labels in clinical and biological applications: I. Theory, Anal. Biochem., 1998, 262, 137156.

29 J. Yguerabide and E. E. Yguerabide, Light-scattering submicroscopic particles as highly fluorescent analogs and their use as tracer labels in clinical and biological applications: II. Experimental characterization, Anal. Biochem., 1998, 262, 157-176.

30. G. T. Hermanson. Bioconjugate Techniques (Academic Press, 1996), chapter 14.

31 J. C. Love, L. A. Estroff, J. K. Kriebel, R. G. Nuzzo and G. M. Whitesides, Self-assembled monolayers of thiolates on metals as a form of nanotechnology, Chem. Rev., 2005, 105, 1103-1169.

32 N. Nerambourg, R. Praho, M. H. V. Werts, D. Thomas and M. Blanchard-Desce, Hydrophilic monolayerprotected gold nanoparticles and their functionalisation with fluorescent chromophores, Int. J. Nanotechnol., 2008, 5, 722-740.

33 R. Samy, T. Glawdel and C. L. Ren, Method for microfluidic whole-chip temperature measurement using thinfilm poly(dimethylsiloxane)/rhodamine B, Anal. Chem., 2008, 80, 369-375.

34 B. E. Bayer, Color imaging array, U. S. Patent, 1976, US 3,971,065.

35 A. D. Stroock, S. K. W. Dertinger, A. Ajdari, I. Mezić, H. A. Stone and G. M. Whitesides, Chaotic mixer for microchannels, Science, 2002, 295, $647-651$.

36. P. Tabeling. Introduction to microfluidics (Oxford University Press, Oxford, UK, 2005), chapter 3, p. 142.

37 N. L. Jeon, S. K. W. Dertinger, D. T. Chiu, I. S. Choi, A. D. Stroock and G. M. Whitesides, Generation of Solution and Surface Gradients Using Microfluidic Systems, Langmuir, 2000, 16, 8311-8316.

38 M. Bonn, H. J. Bakker, G. Rago, F. Pouzy, J. R. Siekierzycka, A. M. Brouwer and D. Bonn, Suppression of proton mobility by hydrophobic hydration, J. Am. Chem. Soc., 2009, 131, 17070-17071.

39 C. N. Baroud, F. Okkels, L. Ménétrier and P. Tabeling, Reaction-diffusion dynamics: Confrontation between theory and experiment in a microfluidic reactor, Phys. Rev. E, 2003, 67, 060104.

40 Y. Wang, T. Mukherjee and Q. Lin, Systematic modeling of microfluidic concentration gradient generators, J. Micromech. Microeng., 2006, 16, 2128-2137.

41 P. Galambos and F. K. Forster, Micro-fluidic diffusion coefficient measurement, $\mu$ TAS, 1998, 189-191.

42 R. K. Jain, Transport of macromolecules in tumor microcirculation, Biotechnol. Prog., 1985, 1, 81-94.

43 M. Loumaigne, R. Praho, D. Nutarelli, M. H. V. Werts and A. Débarre, Fluorescence correlation spectroscopy reveals strong fluorescence quenching of FITC adducts on PEGylated gold nanoparticles in water and the presence of fluorescent aggregates of desorbed thiolate ligands, Phys. Chem. Chem. Phys., 2010, 12, 1100411014.

44 N. L. Jeon, H. Baskaran, S. K. W. Dertinger, G. M. Whitesides, L. Van De Water and M. Toner, Neutrophil chemotaxis in linear and complex gradients of interleukin-8 formed in a microfabricated device, Nat. Biotech., 2002, 20, 826-830.

45 A. Tirella, M. Marano, F. Vozzi and A. Ahluwalia, A microfluidic gradient maker for toxicity testing of bupivacaine and lidocaine, Toxicol. in Vitro, 2008, 22, 1957-1964.

46 D. Quinton, A. Girard, L. To Thi Kim, V. Raimbault, L. Griscom, F. Razan, S. Griveau and F. Bedioui , On-chip multi-electrochemical sensor array platform for simultaneous screening of nitric oxide and peroxynitrite, Lab Chip, 2011, 11, 1342-1350.

47 J. Turkevich, P. C. Stevenson and J. Hillier, A study of the nucleation and growth process in the synthesis of colloidal gold, Discuss. Faraday Soc., 1951, 11, 55-75.

48 G. Frens, Controlled nucleation for the regulation of the particle size in monodisperse gold suspensions, Nature Phys. Sci., 1973, 241, 20-22.

49 J. R. G. Navarro, M. Plugge, M. Loumaigne, A. Sanchez Gonzalez, B. Mennucci, A. Débarre, A. M. Brouwer and M. H. V. Werts, Probing the interactions between disulfide based ligands and gold nanoparticles using a functionalised fluorescent perylene-monoimide dye, Photochem. Photobiol. Sci., 2010, 9, 1042-1054. 